\title{
Removal of ECG artifacts from EMG signals with different artifact magnitudes by template subtraction
}

https://doi.org/10.1515/cdbme-2019-0090

\begin{abstract}
This work investigates the performance of eventsynchronous noise cancelling algorithms to separate ECG artifacts from thoracic EMG recordings. In focus are the precise detection of heart beats, the exact time alignment of the QRS segments and the construction of a template QRS signal. Among the utilized methods is a modified structural intensity (SI) approach based on the observation of extrema in low pass filtered versions of the second derivative as a detector. An adoption of this approach is also used to obtain the exact time alignment of QRS segments. Artificial test signals are constructed based on addition of ECG and sEMG with different magnitudes. We show that approaches based on the observation of extrema in different scales yield superior results.
\end{abstract}

Keywords: ECG, sEMG, template subtraction, eventsynchronous interference cancelling, structural intensity

\section{Introduction}

Analysis of surface electromyography (sEMG) can play a helpful role in gaining information about the state of muscles in a noninvasive way. However, surface EMG recordings from muscles close to the heart are often contaminated by cardiogenic disturbances. Methods based on adaptive noise cancelling were proposed to isolate the EMG and the ECG part of the signal. Strobach et al. [8] proposed a method that utilizes the repetitive nature of the cardiogenic artifacts. A template QRS segment is estimated by event-synchronous averaging of the contaminated signal. The cardiogenic artifacts are canceled by time-synchronously subtracting this template from the contaminated signal. A theoretical analysis of the error induced in case of such an artificial noise signal was carried out by Ungureanu \& Wolf [9]. The key components of such an eventsynchronous interference canceller are (1) the QRS detector, (2) the determination of the exact time alignment and (3) the construction of the template. This work evaluated the perfor-

*Corresponding author: Lorenz Kahl, Drägerwerk AG \& Co. KGaA, Moislinger Allee 53-55, 23558 Lübeck, e-mail: lorenz.kahl@draeger.com Ulrich G. Hofmann, Section for Neuroelectronic Systems, Faculty of Medicine, University of Freiburg, Engesserstraße 4, 79108 Freiburg, e-mail: ulrich.hofmann@coregen.uni-freiburg.de mance of the three key components based on artificial test signals. The focus was put on the ability of the applied algorithms to cope with increased EMG magnitudes.

\section{Methods}

Our adaptive noise canceller performed the event synchronous averaging and subtraction with sample rate $f_{s}=2048 \mathrm{~Hz}$. This is close to the $2 \mathrm{kHz}$ used by Guméry et al. [3] and enables time alignment with a resolution of approximately 0.5 ms.

\subsection{QRS Detection}

Guméry et al. [3] described a new approach for detecting the QRS segments based on the modified structural intensity. This method is based on the observation of extrema in a derivative of the signal that is low pass filtered into different scales (Fig. 1). In contrast to the original SI based method we processed only a limited number of scales. We used powers of two and a further intermediate scale in-between leading to 28 scales $N \in\left\{2^{\alpha}: 0 \leq \alpha \leq 14\right\} \cup\left\{3 \cdot 2^{\beta}: 0 \leq \beta \leq 12\right\}$. The filtered signal for each scale was obtained by a moving average filter. The filter coefficients were based on the convolution of a part responsible for the derivation $([1,-2,1]$ as $r=2)$ and the Nth row of Pascal's triangle responsible for low pass filtering.

Corresponding extrema over different scales were aligned to yield a set $Q_{r}$ of extrema lines. These lines consisted of up to 29 extrema corresponding to the 2 nd derivative signal (as $r=2$ ) as scale 0 and the 28 low pass filtered versions. $G_{m}^{* * *}$ is calculated based on the set of extrema lines $Q_{2}$ and features peaks corresponding to every QRS segment.

$$
G_{m}^{* * *}(t)=\frac{1}{\epsilon_{G}} \sum_{i \in Q_{2}} \sum_{N=N_{m i n}}^{N_{\max }} h(i, N) \cdot \theta\left(\frac{t-\tau_{2}(i, 0)}{\frac{1}{4} \cdot \frac{176}{2000}}\right)
$$

with $h(i, N)=\frac{\left|s_{2}(i, N)\right|}{(N+1)^{r+1 / 2}} \cdot \tau_{r}(i, N)$ and $s_{r}(i, N)$ denote the time and the value of the $i$ th extrema line at scale $N$ and derivative order $r . \theta$ is the Gaussian function, $\epsilon_{G}$ is used to normalize the values, $N_{\min }=8192$ and $N_{\max }=16384$ 


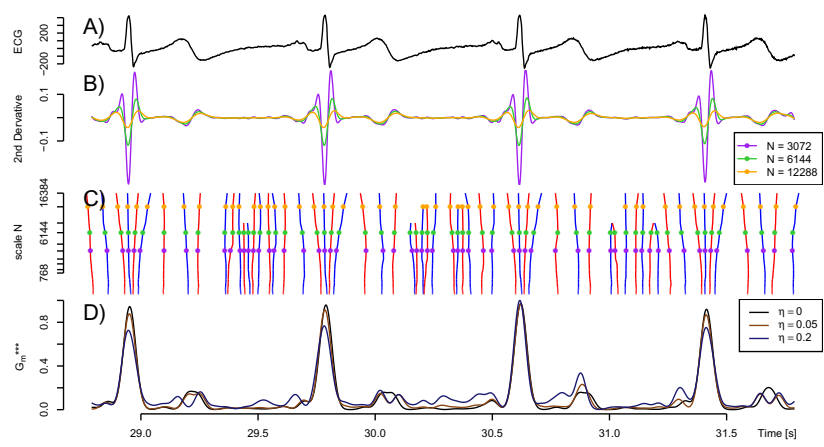

Fig. 1: Structural intensity based QRS detection. A) shows a segment of the pure ECG signal $(\eta=0)$ while $\mathrm{B})$ shows differently low pass filtered scales of the 2 nd derivative. C) visualizes the extrema lines from $Q_{2}$. The maxima and minima lines are drawn in red and blue, respectively. The thickness of the line corresponds to the magnitude of the extremum. The colored dots correspond to the extrema of the scale in the same color in B). D) shows the resulting $G_{m}^{* * *}$ signal. Additionally the resulting $G_{m}^{* * *}$ based on input with different EMG levels $\eta=0.05,0.2$ are shown.

For comparison purposes a classical QRS detector was also used. This traditional QRS detection was performed by the EP Limited software [4]. It is similar to the algorithm suggested by Pan \& Tompkins [6].

\subsection{Exact Time Alignment of QRS}

The determination of exact time locations is also based on the idea to track extrema in low pass filtered signals. Next to the second derivative used for detection we also included the nonderived signal and its first derivative. A preliminary mean heartbeat was calculated by superimposing the QRS segments based on the coarse time locations from the QRS detector. This preliminary template beat was subject to construction of reference extrema line sets $R_{0}, R_{1}$ and $R_{2}$ covering only the one mean heartbeat, based on the signal itself and its first and second derivatives. The construction was analogous to the extrema line set $Q_{2}$ in the SI based QRS detector. For the exact time alignment the extrema line sets were separated by the extrema type. $Q_{r}^{+}$and $R_{r}^{+}$consisted of the maxima lines whereas $Q_{r}^{-}$and $R_{r}^{-}$held the minima lines.

The magnitude, time location and type of extrema in certain scales can be used as a finger print for a QRS segment. A rating function $E_{r}(t, N)$ determinated the matching of the extrema set $Q_{r}$ in the proximity of time $t$ to the reference extrema set $R_{r}$ based on scale $N$. Large peaks of this rating function correspond to the exact time locations of the QRS segments. The matching is processed separately for minima and maxima and multiplied to yield the combined rating function $E_{r}(t, N)=E_{r}^{+}(t, N) \cdot E_{r}^{-}(t, N)$. The value at a given time

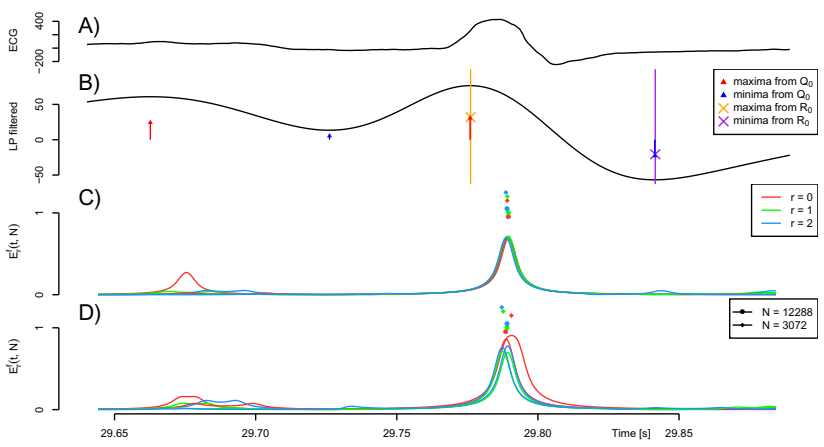

Fig. 2: Exact Time Alignment of QRS. A) shows an exemplary segment from the input signal. $\ln B$ ) the low pass filtered signal at scale $N=12288$ and derivative order $r=0$ (no derivation) is shown. Its maxima and minima are shown in red and blue, respectively. Furthermore the maxima and minima from the reference set $R_{r}$ placed at a position corresponding to the optimal time alignment are shown in orange and purple. $\mathrm{C}$ ) shows the alignment rating functions $E_{r}^{f}(t, N)$ for $r=0,1,2$ and $N=3072,12288$ based on the segment from A). D) depicts the rating functions for the same ECG segment with higher EMG contamination $(\eta=0.2)$. In both $\mathrm{C}$ ) and D) the colored dots visualize the exact time alignment based on different scales and derivative orders.

$t$ is a sum over all extrema in the reference set $R_{r}$. For each extrema a product of $\phi_{t}$ and $\phi_{s}$ quantifies how good the closest extrema in $Q_{r}$ fits in time and magnitude, respectively.

$$
E_{r}^{+}(t, N)=\frac{1}{\epsilon_{E}} \sum_{i \in R_{r}^{+}} \phi_{s}(i, t, N, r) \cdot \phi_{t}(i, t, N, r)
$$

Regarding the minima $E_{r}^{-}(t, N)$ is defined correspondingly.

To calculate the value of the rating function at time $t$ the extrema of the reference set $R_{r}$ are placed centered at $t$. For each extrema $i$ from the reference set $R_{r}$ the closest partner $j$ of the same type from $Q_{r}$ is determined. The distance in time between both extrema is evaluated to yield $\phi_{t}(i, t, N, r)=$ $\left(1+250\left|\tau_{r}^{R}(i, N)-\tau_{r}^{Q}(j, N)\right|\right)^{-2}$.

The magnitudes $s_{r}^{R}$ and $s_{r}^{Q}$ of both partner extrema is incorporated into the factor $\phi_{s}(i, t, N, r)=$ $\left|\epsilon_{Q} \cdot s_{r}^{Q}(j, N)\right|^{2}\left(1+\frac{\left|\epsilon_{Q} \cdot s_{r}^{Q}(j, N)\right|}{\left|\epsilon_{R} \cdot s_{r}^{R}(i, N)\right|}\right)^{-2}$. The factor yields highest values if both partnered items have the same magnitude and lower values otherwise. Furthermore it assures that the larger magnitudes from the reference set (corresponding to the most dominant features in the QRS) are given the higher weights. Applied scalings are again denoted by $\epsilon$.

The exact time location for each heartbeat coarsely detected at time $b_{i}$ is calculated as $y_{i}(r, N)=$ $\max _{b_{i}-\Delta \leq t \leq b_{i}+\Delta} E_{r}^{f}(t, N) . E_{r}^{f}(t, N)$ is obtained by filtering $E_{r}(t, N)$ with a blackman window with $n=20$. In case of low EMG levels the $y_{i}$ based on different scales $N$ and derivative order $r$ fall very close together. However, in cases with elevated EMG levels it turned out to be reasonable to 
combine the exact time locations from different scales and orders of derivation. We propose to use $z_{i}=\operatorname{median}\left\{y_{i}(r, N)\right\}$ for $r=0,1,2$ and $N=3072,12288$. Fig. 2 visualizes this method (DerivativeExtrema).

A second approach (Flank) calculated the exact time location by threshold intersection within the strongest slope of the QRS. For each recording it was determined whether the strongest flank within QRS range was rising or falling and which threshold value best detected this flank by intersection. The exact time alignment for each QRS was determined by intersection of the largest flank with this threshold.

Another method (ShiftOpt) is based on the idea to regard the alignment as an optimization problem. The signal traces were shifted by integer multiples of the sampling rate so that the mean squared error is minimized. In a preparatory step all QRS segments from the detection were aligned to each other. To reduce the complexity of the n-by-n optimization it was done in groups of seven segments. Each group was reduced to a single segment by averaging after being aligned internally. This procedure was repeated until all segments were reduced into one reference segment. To determine the exact time alignment the individual segments from the ECG signal were aligned to this reference segment.

\subsection{Calculation of the template}

Based on the exact time alignments a template heartbeat segment was calculated by event synchronously averaging all heartbeats. An alternative template was calculated by using the median instead of mean to combine values at the same relative position. For both variants it was investigated whether an additional Savitzky Golay [7] filtering (order 6 and length 25) improves the template.

\subsection{Evaluation with Artificial Signals}

Artificial test signals were constructed to establish a quantitative comparison of the different methods. Six test signals (each 60s long) were constructed based on ECG with additive EMG components of different magnitude (Fig. 3). The magnitude was quantified by an EMG level $\eta$ defined as the quotient of RMS EMG magnitude and peak-to-peak QRS amplitude. Test signals were generated for EMG levels $\eta=0,0.02,0.05,0.1,0.2$. ECG records were obtained from CARDIODAT [1] via Physionet [2]. Channel I was interpolated from $1 \mathrm{kHz}$ to $1024 \mathrm{~Hz}$ to match the sEMG sample rate. Surface EMG recordings from contractions of the upper arm with $60 \%$ MVC level are taken from a previous study [5]. In a preparatory step the input signals were high pass filtered with

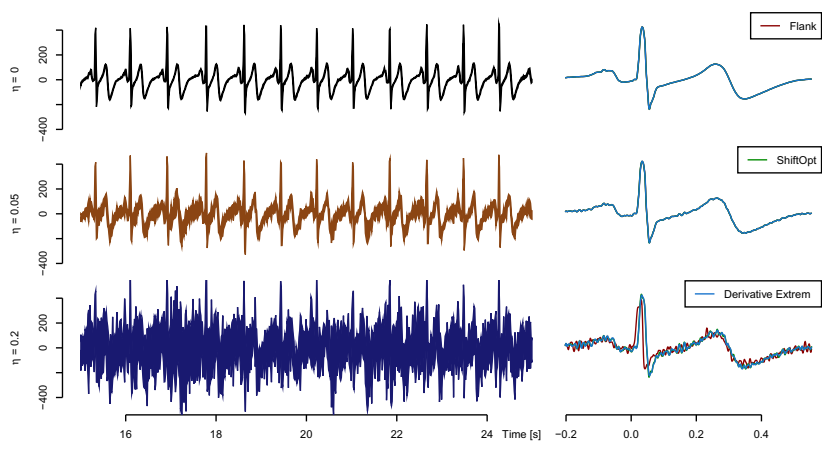

Fig. 3: Artificial Signals. Exemplary artificial test signals with EMG levels $\eta=0,0.05,0.2$ are shown on the left. The resulting heartbeat templates are shown to the right. Their colors correspond to the colors in Fig 4. While they are hardly distinguishable in low EMG situations, they clearly differ in case of higher EMG levels.

a Butterworth filter (third order, cut off frequency $1 \mathrm{~Hz}$ ). Furthermore the input signals were upsampled by a factor of two to match the sample rate for the noise cancelling.

In the subsequent performance evaluation the results based on the pure ECG signal without EMG were taken as target. The different methods are evaluated in respect to their characteristics to yield constant results despite higher EMG levels. This reflects the desired properties to detect, time align and subtract heartbeat artifacts also in applications with EMG noise.

\section{Results}

Regarding detection the structural intensity (SI) based approach showed a better performance than the classical EPlimited detector. Except in $2 \%$ of the cases at $\eta=0.2$ the SI methods detected the QRS despite EMG noise. The EP detector failed to detect the QRS at $\eta=0.1,0.2$ at $7 \%$ and $38 \%$ of the cases, respectively.

In respect to the detection of the exact time location the jitter of the time differences between the zero EMG case and each EMG magnitude is evaluated (Fig. 4). The derivative extrema approach showed slightly better results as the obtained by shifting and error minimization. However, both approaches can clearly improve the quality of time alignment compared to the SI based detector. The results of a detector based on flank threshold intersection showed a poorer performance especially at larger EMG magnitudes.

Already the ECG signals without artificially added EMG contain small amounts of EMG and other noises. Therefore it is not directly assessable whether the ECG was canceled perfectly by expecting the signal to be filtered out completely in case of no EMG. The same holds if one would expect that the 


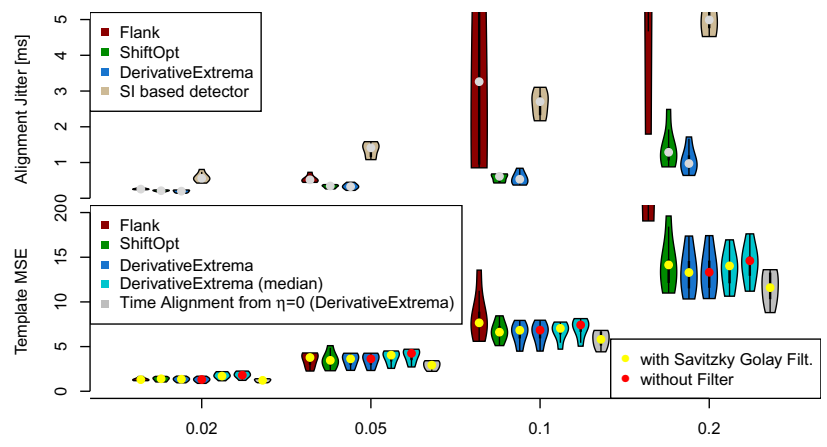

Fig. 4: Results. The first panel shows the jitter of the time alignment relative to the times of the pure ECG signal depending on the EMG level $\eta$. The second panel shows the mean squared error between templates constructed from the pure ECG signal (without filter) and templates (with/without Savitzky Golay filter) constructed from signals with artificially added EMG noise. Except otherwise noted the templates are based on the mean.

filtered out ECG at the higher load levels is equal to the original ECG signal. To evaluate the performance of the template subtraction nevertheless we decided to evaluate the template. In case of a perfect working algorithm the template would be invariant in respect to higher EMG levels. In this case the template would not induce any further disturbances arising from EMG noise in the subtraction process. To exclude bias from Savitzky Golay filtering, the templates from higher EMG levels were compared to unfiltered templates from pure ECG.

The different algorithm's ability to construct this invariant template over the different load levels is correlated with the ability to find the right time. Again the DerivativeExtrema algorithm yielded good results especially in case of higher EMG loads. Applying the mean yielded better results compared to median regarding the construction of the template based on the aligned segments. The Savitzky Golay filter improved the template in case of median but is still behind the mean template. In case of mean filtering had virtually no effect.

\section{Conclusion}

The results clarify that it is reasonable to separate the tasks of detecting the QRS segments and finding their exact time for event synchronous ECG noise cancelling. Algorithms that are specifically designed to find the exact time location are far less prone to misalignment caused by EMG noise.

The newly introduced Derivative Extrema method shows a performance comparable to ShiftOpt and outperforms it in case of higher EMG loads. The reason might be that it focuses more effectively on cardiogenic characteristics in the signal at higher EMG loads. ShiftOpt would also optimize in oscilla- tions that are not cardiogenic but arise from the limited sample size not allowing for the EMG to cancel out completely. The consideration of threshold intersection is only usable as the amplitude of the EMG is less than the QRS amplitude. It might be assumed that this is also the case for other algorithms that rely on single points in the signal. The application of the median could not improve the template. An explanation might be that the median tends to jump and not change smoothly introducing some high frequency disturbance that even a downstream filter cannot completely compensate.

This work was limited to static contractions and regular ECG activity. Further investigation is necessary to include other effects like cyclic EMG activity or irregularities in the ECG signal itself.

\section{Author Statement}

Research funding: The author state no funding involved. Conflict of interest: Authors state no conflict of interest. Informed consent: Informed consent has been obtained from all individuals included in this study. Ethical approval: The research related to human use complies with all the relevant national regulations, institutional policies and was performed in accordance with the tenets of the Helsinki Declaration, and has been approved by the authors' institutional review board or equivalent committee.

\section{References}

[1] Bousseljot R, Kreiseler D et al. Nutzung der EKG-Signaldatenbank CARDIODAT der PTB über das Internet. Biomedizinische Technik/Biomedical Engineering 317-318, 1995.

[2] Goldberger AL, Amaral LAN et al. PhysioBank, PhysioToolkit, and PhysioNet. Circulation 101(23):e215-e220, 2000.

[3] Guméry PY, Fontecave-Jallon J et al. Modified structural intensity for singularity localization in noisy signals: Application to coherent averaging for event-synchronous ECG interference cancellation in diaphragmatic EMG signals. Int J Adapt Control Signal Process 24(5):424-433, 2009.

[4] Hamilton PS. Open Source ECG Analysis Software Documentation. Tech. rep., E.P. Limited, 2002.

[5] Kahl L, Eger $\mathrm{M}$ et al. Effects of sampling rate on automated fatigue recognition in surface EMG signals. Current Directions in Biomedical Engineering 1(1):80-84, 2015.

[6] Pan J, Tompkins WJ. A Real-Time QRS Detection Algorithm. IEEE Trans Biomed Eng BME-32(3):230-236, 1985.

[7] Savitzky A, Golay MJE. Smoothing and Differentiation of Data by Simplified Least Squares Procedures. Anal Chem 36(8):1627-1639, 1964.

[8] Strobach P, Abraham-Fuchs K et al. Event-synchronous cancellation of the heart interference in biomedical signals. IEEE Trans Biomed Eng 41(4):343-350, 1994.

[9] Ungureanu M, Wolf W. Basic Aspects Concerning the EventSynchronous Interference Canceller. IEEE Trans Biomed Eng 53(11):2240-2247, 2006. 